BULL. AUSTRAL. MATH. SOC.

\title{
A CHARACTERIZATION OF RECURRENT MOTIONS
}

\author{
RONALD A. KNIGHT
}

Necessary and sufficient conditions for a Poisson stable flow to be recurrent are given.

An elegant necessary and sufficient condition for a flow on a locally compact phase space to be recurrent was reported in [4], [1]. Unfortunately, however, the condition has proven to be necessary but not sufficient. In this note we recall a counterexample and give a necessary and sufficient condition. We use the notations and definitions of [1] and [4]. Throughout the note we let $(X, \pi)$ denote a given flow or dynamical system on a Hausdorff phase space $X$. We shall say that a set $M \subset X$ (point $x \in X$ ) is approximated by weak attractors relative to an invariant set $H \subset X$ provided each neighborhood $U$ of $M$ (of $x$ ) contains a weak attractor neighborhood $V$ of $M$ relative to the subflow $(H, \pi \mid H)$. Whenever we say that $M$ (or $x$ ) is approximated by weak attractors of a property $P$ relative to $H$, we mean that there exists at least one neighborhood $V$ of property $P$ satisfying the requirements of the preceding definition. Before stating and proving our theorem we note that the proof of Theorem $I$ in [4] is correct except for a rather subtle tacit incorrect assumption. Our proof uses a somewhat similar approach.

THEOREM A. A (positively, negatively) bilaterally Poisson stable flow on a locally compact space $X$ is recurrent if and only if each point $x$ of $X$ is approximated by compact (positive, negative) weak attractors relative to its orbit closure $K(x)$.

Proof. Let $(X, \pi)$ be bilaterally Poisson stable and let $x$ be a

Received 21 February 1983. 
pcint of $X$. We shall proceed by showing that $x \in L^{+}(y)$ for each point $y \in L^{+}(x)$ whenever each point is approximated by compact weak attractors relative to its orbit closure. Suppose the contrary; that is, for some point $y$ of $L^{+}(x)$ we have $x \leqslant L^{+}(y)$. Throughout the remainder of this paragraph we argue relative to the subspace $Y=L^{+}(x)$ of $X$. In $Y$ select relatively compact open neighborhoods $V, U$, and $W$ of $x$ such. that $\bar{V} \subset U, \bar{U} \subset W$, and $K(y)=L^{+}(y) \subset y \backslash \bar{W}$. The neighborhood $\bar{W}$ can be chosen so that it contains no complete trajectory or semitrajectory. Also, we can choose $\bar{V}$ to be a compact weak attractor and choose $\bar{U} \subset A_{w}^{+}(\bar{V})$. Let

$$
t_{z}=\inf \left\{t \in R^{+}: z t \in U \text { and } z[0, t] \cap(Y \backslash \bar{U}) \neq \emptyset\right\}
$$

for each point $z \in \bar{U}$. Each point $z \in \bar{U}$ has a neighborhood $U_{z} \subset A_{w}^{+}(\bar{V})$ such that $U_{z} t \subset Y \backslash \bar{U}$ and $U_{z} t^{\prime} \subset U$ for some $t$ and $t^{\prime}$ with $0<t<t^{\prime}$ because $\pi$ is continuous and $z$ is bilaterally Poisson stable. Moreover, we can select $t^{\prime}$ so that $t_{p} \leq t^{\prime}<t_{z}+1$ for each $p \in U_{z} \cap \bar{U}$. Since $\bar{U}$ is compact and $\left\{U_{z}: z \in \bar{U}\right\}$ covers $\bar{U}$, there is a finite subcover $\left\{U_{z_{1}}, \ldots, U_{z_{n}}\right\}$ for $\bar{U}$. Thus

$$
T=\sup \left\{t_{z}: z \in \bar{U}\right\} \leq \max \left\{t_{z_{i}}+1: i=1, \ldots, n\right\}<+\infty \text {. }
$$

Note that $C(U)=U \cup U[0, T]$. Now select a net $\left(t_{i}\right)$ such that $x t_{i} \rightarrow y$ as $t_{i} \rightarrow+\infty$. Let

$$
T_{i}=\max \left\{t \in R^{+}: x t \in \partial U \text { and } t \leq t_{i}\right\} \text {. }
$$

Some subnet $\left(x T_{n_{i}}\right)$ of $\left(x T_{i}\right)$ converges to a point $z$ in the compact set $\partial U$. Untimately, the net $\left(x t_{n_{i}}\right)$ is in $Y \backslash \bar{U}$, and hence, for some $i_{0}$ we have $x\left(T_{n_{i}}, t_{n_{i}}\right] \subset y \backslash \bar{U}$ for $i \geq i_{0}$. Also

$$
x t_{n_{i}}=\left(x_{n_{i}}\right)\left(t_{n_{i}}{ }^{-T}{ }_{n_{i}}\right) \in(\partial U)[0, T]
$$

for $i \geq i_{0}$ which yields $y \in(\partial U)[0, T]$. Thus $C^{-}(y) \cap \bar{U} \neq \emptyset$ contradicting $C(y) \subset K(y)=L^{+}(y) \subset y \backslash \bar{U}$. Whence we have $x \in L^{+}(y)$ and 
therefore $L^{+}(y)=L^{+}(x)$.

Each point of $L^{+}(x)$ is weakly attracted to $x$. As noted in [4] each point of $K(x)=L^{+}(x)$ is weakly attracted to $x$ if and only if $K(x)$ is compact minimal. Thus $L^{+}(x)$ is compact minimal for each $x \in X$. Since each point of a compact minimal set is recurrent, $X$ is recurrent.

Conversely, each recurrent point is bilaterally Poisson stable [4], [3, 4.10.2]. For any point $x \in X$, each point of $K(x)$ is weakly attracted to $x$, and hence, each point of $K(x)$ is weakly attracted to every compact neighborhood of $x$ relative to $K(x)$. Consequently, each point of $X$ is approximated by compact weak attractors relative to its trajectory closure completing the proof.

The pairwise equivalence of $(a),(b),(c)$, and (d) of the following corollary is known (see [4]).

COROLLARY B. Let $X$ be locally compact. Then the following are pairwise equivalent for a closed invariant set $M$ :

(a) $M$ is compact minimal;

(b) $M$ is compact positively (negatively) minimal;

(c) $M$ is an orbit closure, each point of which is recurrent;

(d) $M$ is an orbit closure, each point of which weakly attracts $M$; and

(e) $M$ is a (positively, negatively) bilaterally Poisson stable orbit closure, each point of which is approximated by compact (positive, negative) weak attractors relative to $M$.

COROLLARY $C$. Let $X$ be locally compact. The nonwondering points are recurrent if and only if the set of bilaterally Poisson stable points is closed and each bilaterally Poisson stable point is approximated by compact weak attractors relative to its orbit closure.

Gottschalk's example [3, p. 764] of a nonrecurrent bilaterally Poisson stable flow on a compact metric space shows that Poisson stability alone is not sufficient in Theorem A. It consists of a subflow of the Bebutoff flow formed by the orbit closure of a bounded uniformly continuous function $f$ 
constructed by Bohr [2, pp. 113-117]. The function $f$ has arbitrarily long intervals on which $f$ has value zero. The zero function is the only recurrent point of $K(f)$, the only point approximated by compact weak attractors, and the only point weakly attracting $K(f)$.

\section{References}

[1] Shair Ahmad, "Strong attraction and classification of certain continuous flows", Math. Systems Theory 5 (1971), 157-163.

[2] Harald Bohr, "Zur Theorie der fast periodischen Funktionen", Acta Math. 45 (1924), 29-127.

[3] W.H. Gottschalk, "Almost periodic points with respect to transformation semi-groups", Ann. of Math. (2) 47 (1946), 762-766.

[4] Ronald A. Knight, "Recurrent and Poisson stable flows", Rroc. Amer. Wath. Soc. 83 (1981), 49-53.

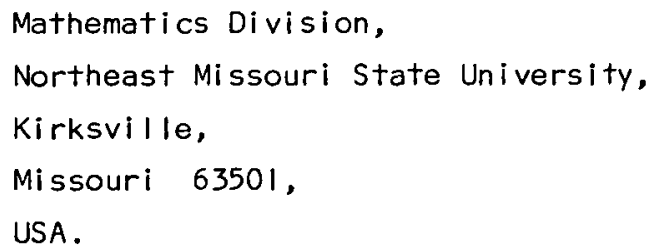

